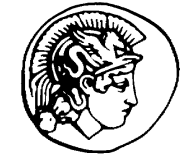

PERGAMON
science@Directo

International Journal of Non-Linear Mechanics 38 (2003) 1133-1148
NON-LINEAR

MECHANICS

www.elsevier.com/locate/ijnonlinmec

\title{
First-passage failure and its feedback minimization of quasi-partially integrable Hamiltonian systems
}

\author{
W.Q. Zhu ${ }^{\mathrm{a}, \mathrm{b}, *}$, Z.L. Huang ${ }^{\mathrm{a}}$, M.L. Deng ${ }^{\mathrm{a}}$ \\ a Department of Mechanics, Zhejiang University, Hangzhou 310027, People's Republic of China \\ ${ }^{\mathrm{b}}$ State Key Laboratory of Nonlinear Mechanics, Institute of Mechanics, Chinese Academy of Sciences, \\ Beijing 100080, People's Republic of China
}

\begin{abstract}
An $n$ degree-of-freedom Hamiltonian system with $r(1<r<n)$ independent first integrals which are in involution is called partially integrable Hamiltonian system. A partially integrable Hamiltonian system subject to light dampings and weak stochastic excitations is called quasi-partially integrable Hamiltonian system. In the present paper, the procedures for studying the first-passage failure and its feedback minimization of quasi-partially integrable Hamiltonian systems are proposed. First, the stochastic averaging method for quasi-partially integrable Hamiltonian systems is briefly reviewed. Then, based on the averaged Itô equations, a backward Kolmogorov equation governing the conditional reliability function, a set of generalized Pontryagin equations governing the conditional moments of first-passage time and their boundary and initial conditions are established. After that, the dynamical programming equations and their associated boundary and final time conditions for the control problems of maximization of reliability and of maximization of mean first-passage time are formulated. The relationship between the backward Kolmogorov equation and the dynamical programming equation for reliability maximization, and that between the Pontryagin equation and the dynamical programming equation for maximization of mean first-passage time are discussed. Finally, an example is worked out to illustrate the proposed procedures and the effectiveness of feedback control in reducing first-passage failure.(C) 2002 Elsevier Science Ltd. All rights reserved.
\end{abstract}

Keywords: Non-linear system; Stochastic excitation; Stochastic averaging; First-passage failure; Reliability; First-passage time; Stochastic optimal control; Dynamical programming

\section{Introduction}

First-passage failure (first excursion) is a major failure model of mechanical/structural systems under random excitation and it is among the most difficult problems in the theory of random vibration or stochastic structural dynamics. At present, a mathematical exact solution is possible only if the random

\footnotetext{
* Corresponding author. Tel.: +86-571-799-1150; fax: +86-571-795-1358.

E-mail address: wqzhu@yahoo.com (W.Q. Zhu).
}

phenomenon in question can be treated as a diffusion process. Still, known solutions are limited to the one-dimensional case $[1,2]$.

The state space of a mechanical or structural system model is generally two dimensional or higher and the random excitation of the system is usually not Gaussian white noise. So, it is difficult to directly use the theory of diffusion process for first-passage problem. A feasible way in this case is first to apply the stochastic averaging method to reduce the system to averaged Itô equations. In the last three decades, many researchers applied the 
classical stochastic averaging method to study the first-passage problem of single-degree-of freedom (SDOF) oscillators with linear or non-linear restoring force [3-13]. Recently, Zhu et al. applied the stochastic averaging method for quasi-non-integrable and integralbe Hamiltonian systems [14,15] to study the first-passage problem of many-degree-of-freedom (MDOF) quasi-non-integrable and quasi-integralbe Hamiltonian systems $[16,17]$.

In the mechanical and structural systems, feedback control has been mostly used to alleviate the response and sometimes used to stabilize the systems. The non-linear stochastic optimal control strategy proposed recently by the present first author and his co-worker $[18,19]$ can also be used to reduce the first-passage failure $[20,21]$ except response reduction [22]. In the present paper, the first-passage failure and its feedback minimization of quasi-partially integrable Hamiltonian systems are studied. First, the stochastic averaging method for quasi-partially integralbe Hamiltonian systems developed recently is briefly reviewed. Then, the backward Kolmogorov equation governing the conditional reliability function and the generalized Pontryagin equations governing the conditional moments of first-passage time and their boundary and initial conditions are established from the averaged Ito equations. After that, the dynamical programming equations and their boundary and final time conditions for the control problems of maximization of reliability and of maximization of mean first-passage time are formulated. The optimal control forces are determined. The relationship between the dynamical programming equations and backward Kolmogorov equation and Pontryagin equation for an optimally controlled system are discussed. Finally, an example is given to illustrate the proposed procedures and the effect of control on the reliability and mean first-passage time.

\section{The stochastic averaging method}

Consider an $n$ degree-of-freedom (DOF) quasiHamiltonian system governed by the following equations of motion:

$\dot{Q}_{i}=\frac{\partial H^{\prime}}{\partial P_{i}}$,
$\dot{P}_{i}=-\frac{\partial H^{\prime}}{\partial Q_{i}}-\varepsilon c_{i j} \frac{\partial H^{\prime}}{\partial P_{j}}+\varepsilon^{1 / 2} f_{i k} W_{k}(t)$,

$i, j=1,2, \ldots, n, \quad k=1,2, \ldots, m$,

where $Q_{i}$ and $P_{i}$ are generalized displacements and momenta, respectively, $H^{\prime}=H^{\prime}(\mathbf{Q}, \mathbf{P})$ is a twice differentiable Hamiltonian, $c_{i j}=c_{i j}(\mathbf{Q}, \mathbf{P})$ are functions representing coefficients of quasi-linear dampings; $f_{i k}=f_{i k}(\mathbf{Q}, \mathbf{P})$ are functions representing amplitudes of stochastic excitations, $\varepsilon$ is a small positive parameter, $W_{k}(t)$ are Gaussian white noises in the sense of Stratonovich with correlation functions $E\left[W_{k}(t) W_{l}(t+\tau)\right]=2 D_{k l} \delta(\tau)$.

Eq. (1) can be modelled as Stratonovich stochastic differential equations and then converted to Itô stochastic differential equations by adding WongZakai correction terms $D_{k l} f_{j l} \partial f_{i k} / \partial P_{j}$ in the second equation of Stratonovich equations. Splitting the Wong-Zakai correction terms into conservative part and dissipative part and then combining them with $-\partial H^{\prime} / \partial Q_{i}$ and $-\varepsilon c_{i j} \partial H^{\prime} / \partial P_{j}$, respectively, the following Itô equations can be obtained $[14,15]$ :

$\mathrm{d} Q_{i}=\frac{\partial H}{\partial P_{i}} \mathrm{~d} t$,

$\mathrm{d} P_{i}=-\left(\frac{\partial H}{\partial Q_{i}}+\varepsilon m_{i j} \frac{\partial H}{\partial P_{j}}\right) \mathrm{d} t+\varepsilon^{1 / 2} \sigma_{i k} \mathrm{~d} B_{k}(t)$,

$i, j=1,2, \ldots, n, \quad k=1,2, \ldots, m$,

where $H=H(\mathbf{Q}, \mathbf{P})$ is a modified Hamiltonian, $m_{i j}=$ $m_{i j}(\mathbf{Q}, \mathbf{P})$ are modified coefficients of dampings; $B_{k}(t)$ are independent unit Wiener processes, $\boldsymbol{\sigma} \boldsymbol{\sigma}^{\mathrm{T}}=2 \mathbf{f D f}^{\mathrm{T}}$.

When $\varepsilon=0$, Eq. (2) is reduced to a set of Hamilton equations. A Hamiltonian system with Hamiltonian $H$ can be non-integrable, partially integrable or completely integrable. Here it is assumed that the Hamiltonian system is partially integrable, i.e., the Hamiltonian system has $r(1<r<n)$ independent first integrals which are in involution. Specifically, the Hamiltonian is assumed of the form

$H(\mathbf{q}, \mathbf{p})=\sum_{\eta=1}^{r-1} H_{\eta}\left(\mathbf{q}_{1}, \mathbf{p}_{1}\right)+H_{r}\left(\mathbf{q}_{2}, \mathbf{p}_{2}\right)$,

where $\mathbf{q}_{1}=\left[q_{1}, q_{2}, \ldots, q_{r-1}\right]^{\mathrm{T}}, \mathbf{p}_{1}=\left[p_{1}, p_{2}, \ldots, p_{r-1}\right]^{\mathrm{T}}$, $\mathbf{q}_{2}=\left[q_{r}, q_{r+1}, \ldots, q_{n}\right]^{\mathrm{T}}, \quad \mathbf{p}_{2}=\left[p_{r}, p_{r+1}, \ldots, p_{n}\right]^{\mathrm{T}^{\mathrm{T}}}$; $H_{\eta}$ and $H_{r}$ are the $r$ independent first integrals in 
involution. Eq. (3) implies that the partially integrable Hamiltonian system consists of an integrable part and a non-integrable part. For the integrable part, action-angle variables can be introduced. Thus, Eq. (3) can be re-written as

$H\left(\mathbf{I}, \mathbf{q}_{2}, \mathbf{p}_{2}\right)=\sum_{\eta=1}^{r-1} H_{\eta}\left(I_{\eta}\right)+H_{r}\left(\mathbf{q}_{2}, \mathbf{p}_{2}\right)$.

The integrable part of a partially integrable Hamiltonian system can also be resonant and non-resonant, just as for integrable Hamiltonian system [15]. Here only non-resonant case is considered. For quasi-partially integrable Hamiltonian systems, the averaged Itô equations in the non-resonant case are of the form [23].

$$
\begin{aligned}
& \mathrm{d} I_{\eta}=a_{\eta}\left(\mathbf{I}, H_{r}\right) \mathrm{d} t+\sigma_{\eta k}\left(\mathbf{I}, H_{r}\right) \mathrm{d} \bar{B}_{k}(t), \\
& \mathrm{d} H_{r}=a_{r}\left(\mathbf{I}, H_{r}\right) \mathrm{d} t+\sigma_{r k}\left(\mathbf{I}, H_{r}\right) \mathrm{d} \bar{B}_{k}(t), \\
& \eta=1,2, \ldots, r-1, \quad k=1,2, \ldots, m,
\end{aligned}
$$

where $\bar{B}_{k}(t)$ are independent unit Wiener processes.

$$
\begin{aligned}
a_{\eta} & =a_{\eta}\left(\mathbf{I}, H_{r}\right) \\
& =\varepsilon\left\langle-m_{\eta^{\prime} j} \frac{\partial H}{\partial P_{j}} \frac{\partial I_{\eta}}{\partial P_{\eta^{\prime}}}+D_{k l} f_{\eta^{\prime} k} f_{\eta^{\prime \prime} l} \frac{\partial^{2} I_{\eta}}{\partial P_{\eta^{\prime}} \partial P_{\eta^{\prime \prime}}}\right\rangle \\
a_{r} & =a_{r}\left(\mathbf{I}, H_{r}\right) \\
& =\varepsilon\left\langle-m_{u j} \frac{\partial H}{\partial P_{j}} \frac{\partial H_{r}}{\partial P_{u}}+D_{k l} f_{u k} f_{v l} \frac{\partial^{2} H_{r}}{\partial P_{u} \partial P_{v}}\right\rangle \\
b_{\eta \bar{\eta}} & =b_{\eta \bar{\eta}}\left(\mathbf{I}, H_{r}\right)=\left[\boldsymbol{\sigma} \boldsymbol{\sigma}^{\mathrm{T}}\right]_{\eta \bar{\eta}} \\
& =\varepsilon\left\langle 2 D_{k l} f_{\eta^{\prime} k} f_{\eta^{\prime \prime} l} \frac{\partial I_{\eta} \partial I_{\bar{\eta}}}{\partial P_{\eta^{\prime}} \partial P_{\eta^{\prime \prime}}}\right\rangle \\
b_{\eta r} & =b_{\eta r}\left(\mathbf{I}, H_{r}\right)=\left[\boldsymbol{\sigma} \boldsymbol{\sigma}^{\mathrm{T}}\right]_{\eta r} \\
& =\varepsilon\left\langle 2 D_{k l} f_{\eta^{\prime} k} f_{u l} \frac{\partial I_{\eta} \partial H_{r}}{\partial P_{\eta^{\prime}} \partial P_{u}}\right\rangle, \\
b_{r r} & =b_{r r}\left(\mathbf{I}, H_{r}\right)=\left[\boldsymbol{\sigma} \boldsymbol{\sigma}^{\mathrm{T}}\right]_{r r} \\
& =\varepsilon\left\langle 2 D_{k l} f_{u k} f_{v l} \frac{\partial H_{r} \partial H_{r}}{\partial P_{u} \partial P_{v}}\right\rangle \\
& \langle
\end{aligned}
$$

$$
\begin{aligned}
&\langle\cdot\rangle= \frac{1}{(2 \pi)^{r-1} T\left(H_{r}\right) V_{\Omega_{1}}} \int_{\Omega} \int_{\Omega}^{2 \pi}\left[\cdot / \frac{\partial H_{r}}{\partial p_{r}}\right] \\
& \times \mathrm{d} \boldsymbol{\theta} \mathrm{d} q_{r} \cdots \mathrm{d} q_{n} \mathrm{~d} p_{r+1} \cdots \mathrm{d} p_{n} \\
& T\left(H_{r}\right)= \frac{1}{V_{\Omega_{1}}} \int_{\Omega}\left[1 / \frac{\partial H_{r}}{\partial p_{r}}\right] \\
& \times \mathrm{d} q_{r} \cdots \mathrm{d} q_{n} \mathrm{~d} p_{r+1} \cdots \mathrm{d} p_{n}, \\
& V_{\Omega_{1}}= \int_{\Omega_{1}} \mathrm{~d} q_{r+1} \cdots \mathrm{d} q_{n} \mathrm{~d} p_{r+1} \cdots \mathrm{d} p_{n}, \\
& \Omega=\left\{\left(q_{r}, \ldots, q_{n}, p_{r+1}, \ldots, p_{n}\right) \mid H_{r}\left(q_{r}, \ldots, q_{n}, 0,\right.\right. \\
&\left.\left.p_{r+1}, \ldots, p_{n}\right) \leqslant H_{r}\right\} \\
& \Omega_{1}=\left\{\left(q_{r+1}, \ldots, q_{n}, p_{r+1}, \ldots, p_{n}\right) \mid H_{r}\left(0, q_{r+1}, \ldots, q_{n}, 0,\right.\right. \\
&\left.\left.p_{r+1}, \ldots, p_{n}\right) \leqslant H_{r}\right\}
\end{aligned}
$$

$i, j=1,2, \ldots, n, \quad \eta, \bar{\eta}, \eta^{\prime}, \eta^{\prime \prime}=1,2, \ldots, r-1$,

$k, l=1,2, \ldots, m, \quad u, v=r, r+1, \ldots, n$,

in which $\boldsymbol{\theta}=\left[\theta_{1}, \theta_{2}, \ldots, \theta_{r-1}\right]^{\mathrm{T}}$.

In many case, $H_{\eta}$ are more easy to obtain than $I_{\eta}$. Since $H_{\eta}=H_{\eta}\left(I_{\eta}\right)$, the equations for $I_{\eta}$ in Eq. (4) can be replaced by those for $H_{\eta}$. The later can be obtained from the former by using Itô differential rule. Denote $\mathbf{H}=\left[H_{1}, H_{2}, \ldots, H_{r}\right]^{\mathrm{T}}$. The averaged Itô equations for $\mathbf{H}$ are of the form

$\mathrm{d} H_{\alpha}=a_{\alpha}(\mathbf{H}) \mathrm{d} t+\sigma_{\alpha k}(\mathbf{H}) \mathrm{d} B_{k}(t)$,

$\alpha=1,2, \ldots, r, \quad k=1,2, \ldots, m$,

where $a_{\alpha}$ and $\sigma_{\alpha k}$ can be obtained similarly as $a_{\eta}, a_{r}, b_{\eta k}$ and $b_{r k}$ in Eq. (6).

\section{The first passage failure}

For many mechanical/structural systems, $H_{\eta}$ represent the energies of $r-1$ degrees of freedom of the integrable part while $H_{r}$ the total energy of the non-integrable part. The state of averaged system of a quasi-partially integralbe system varies randomly in 
the $r$-dimensional domain defined by the direct product of the $H_{\eta}$ intervals and $H_{r}$ interval and safety domain $\bar{\Omega}$ is a bounded region with boundary $\Gamma$ within the $r$-dimensional $H_{\eta}$ and $H_{r}$ domain. Suppose that the lower boundary of safety domain for each $H_{\eta}$ and $H_{r}$ is at zero (it is always possible to make so by using coordinate transformation). Then boundary $\Gamma$ consists of $\Gamma_{0}$ (at least one of $H_{\eta}$ and $H_{r}$ vanishes) and critical boundary $\Gamma_{\mathrm{c}}$. The first passage failure occurs when $\mathbf{H}(t)$ crosses $\Gamma_{\mathrm{c}}$ for the first time, and it is characterized by the conditional reliability function, the conditional probability density or conditional moments of first-passage time, where the word "conditional" means under the given initial condition in safety domain.

The conditional reliability function, denoted by $R\left(t, \mid \mathbf{H}_{0}\right)$, is defined as the probability of $\mathbf{H}(t)$ being in safety domain $\bar{\Omega}$ within time interval $(0, t]$ given initial state $\mathbf{H}_{0}=\mathbf{H}(0)$ being in $\bar{\Omega}$, i.e.,

$R\left(t \mid \mathbf{H}_{0}\right)=P\left\{\mathbf{H}(\tau) \in \bar{\Omega}, \tau \in(0, t] \mid \mathbf{H}_{0} \in \bar{\Omega}\right\}$.

It is the integral of the conditional transition probability density in $\bar{\Omega}$, which is the transition probability density of the sample functions that remain in $\bar{\Omega}$ in time interval $(0, t]$. For averaged system, the conditional transition probability density satisfies the backward Kolmogorov equation with drift and diffusion coefficients in Eqs. (7). Thus, the following backward Kolmogorov equation for the conditional reliability function can be derived

$$
\begin{aligned}
\frac{\partial R}{\partial t} & =a_{\alpha}\left(\mathbf{H}_{0}\right) \frac{\partial R}{\partial H_{\alpha 0}}+\frac{1}{2} b_{\alpha \beta}\left(\mathbf{H}_{0}\right) \frac{\partial^{2} R}{\partial H_{\alpha 0} \partial H_{\beta 0}}, \\
\alpha, \beta & =1,2, \ldots, r,
\end{aligned}
$$

where $a_{\alpha}\left(\mathbf{H}_{0}\right)=\left.a_{\alpha}(\mathbf{H})\right|_{\mathbf{H}=\mathbf{H}_{0}}, b_{\alpha \beta}\left(\mathbf{H}_{0}\right)=\sigma_{\alpha k}(\mathbf{H}) \sigma_{k \beta}$ $\left.(\mathbf{H})\right|_{\mathbf{H}=\mathbf{H}_{0}}, a_{\alpha}(\mathbf{H})$ and $\sigma_{\alpha k}(\mathbf{H})$ are defined by Eq. (7). The boundary conditions are

$R\left(t \mid \Gamma_{0}\right)=$ finite,

$$
R\left(t \mid \Gamma_{\mathrm{c}}\right)=0
$$

which imply that $\Gamma_{0}$ is a reflecting boundary while $\Gamma_{\mathrm{c}}$ absorbing boundary. The initial condition is

$R\left(0 \mid \mathbf{H}_{0}\right)=1, \quad \mathbf{H}_{0} \in \bar{\Omega}$,

which implies that the system is initially in safety domain.
The first-passage time $T$ is defined as the time when the system reaches critical boundary $\Gamma_{\mathrm{c}}$ for the first time given $\mathbf{H}_{0}$ being in $\bar{\Omega}$. Noting that the conditional probability of first-passage failure $F\left(t \mid \mathbf{H}_{0}\right)=$ $1-R\left(t \mid \mathbf{H}_{0}\right)$, the conditional probability density of the first-passage time can be obtained from the conditional reliability function as follows:

$$
p\left(T \mid \mathbf{H}_{0}\right)=\left.\frac{-\partial R\left(t \mid \mathbf{H}_{0}\right)}{\partial t}\right|_{t=T} .
$$

The conditional moments of first-passage time are defined as

$\mu_{l}\left(\mathbf{H}_{0}\right)=\int_{0}^{\infty} T^{l} p\left(T \mid \mathbf{H}_{0}\right) \mathrm{d} T$.

The equations governing the conditional moments of first-passage time can be obtained from Eq. (9) in terms of relations (13) and (14) as follows:

$$
\begin{aligned}
& \frac{1}{2} b_{\alpha \beta}\left(\mathbf{H}_{0}\right) \frac{\partial^{2} \mu_{l+1}}{\partial H_{\alpha 0} \partial H_{\beta 0}}+a_{\alpha}\left(\mathbf{H}_{0}\right) \frac{\partial \mu_{l+1}}{\partial H_{\alpha 0}}=-(l+1) \mu_{l}, \\
& \alpha, \beta=1,2, \ldots, r, \quad l=1,2, \ldots .
\end{aligned}
$$

It is easily seen from Eq. (14) that $\mu_{0}=1$. The boundary conditions associated with Eq. (15) are obtained from Eqs. (10) and (11) in terms of Eqs. (13) and (14). They are

$\mu_{l+1}\left(\Gamma_{0}\right)=$ finite,

$\mu_{l+1}\left(\Gamma_{\mathrm{c}}\right)=0$.

Note that both boundary conditions (10) and (16) are qualitative rather than quantitative. They can be made to be quantitative by using Eqs. (9) and (15), respectively, and the limiting behaviors of the drift and diffusion coefficients in Eqs. (9) and (15) at boundary $\Gamma_{0}$. It will be illustrated with the following example.

The conditional reliability function is obtained from solving backward Kolmogorov equation (9) together with boundary conditions (10) and (11) and initial condition (12). The conditional probability density of first-passage time is obtained from the conditional reliability function by using Eq. (13). The conditional moments of first-passage time are obtained either from the conditional probability density of first-passage time by using definition (14) or directly from solving generalized Pontryagin equation (15) together with boundary conditions (16) and (17), recursively. 


\section{Minimization of first-passage failure by control}

Now consider a controlled quasi-partially integrable Hamiltonian system. The equations of motion of the system are of the form

$\dot{Q}_{i}=\frac{\partial H^{\prime}}{\partial P_{i}}$,

$\dot{P}_{i}=-\frac{\partial H^{\prime}}{\partial Q_{i}}-\varepsilon c_{i j} \frac{\partial H^{\prime}}{\partial P_{j}}+\varepsilon u_{i}+\varepsilon^{1 / 2} f_{i k} W_{k}(t)$,

$Q_{i}(0)=Q_{i 0}, \quad P_{i}(0)=P_{i 0}$,

$i, j=1,2, \ldots, n, \quad k=1,2, \ldots, m$,

where $u_{i}=u_{i}(\mathbf{Q}, \mathbf{P})$ represent feedback control forces and the other notations are the same as those in Eq. (1). Applying the stochastic averaging method for quasi-partially integrable systems described in the last section of Eq. (18), the following averaged Itô equations can be obtained:

$\mathrm{d} H_{\alpha}=\left[a_{\alpha}(\mathbf{H})+\varepsilon\left\langle u_{i} \frac{\partial H_{\alpha}}{\partial P_{i}}\right\rangle\right] \mathrm{d} t+\sigma_{\alpha k}(\mathbf{H}) \mathrm{d} B_{k}(t)$,

$\alpha=1,2, \ldots, r, \quad k=1,2, \ldots, m$,

where $a_{\alpha}$ and $\sigma_{\alpha k}$ are the same as those in Eq. (7) and $\mathbf{H}=\mathbf{H}(t, \mathbf{u})$ is the vector of first integrals of controlled system. For the control problem of maximization of reliability, the value function is defined as

$V\left(t_{1}, \mathbf{H}\right)=\sup _{\mathbf{u} \in U} P\left\{\mathbf{H}(\tau, \mathbf{u}) \in \bar{\Omega}, \tau \in\left[t_{1}, t_{\mathrm{f}}\right]\right\}$,

where $\mathbf{u} \in U$ represents control constraint; "sup" is the abbreviation of word "supremum". Comparison between Eqs. (8) and (20) implies that $V\left(t_{1}, \mathbf{H}\right)$ is the reliability function of optimally controlled system with $t_{1}$ as initial time and under control constraint $\mathbf{u} \in U$. Based on the dynamical programming principle [24], the following stochastic dynamical programming equation for the value function can be derived from the averaged system (19):

$$
\begin{gathered}
\sup _{\mathbf{u} \in U}\left\{\frac{\partial}{\partial t_{1}}+\left[a_{\alpha}(\mathbf{H})+\varepsilon\left\langle u_{i} \frac{\partial H_{\alpha}}{\partial P_{i}}\right\rangle\right] \frac{\partial}{\partial H_{\alpha}}\right. \\
\left.+\frac{1}{2} b_{\alpha \beta}(\mathbf{H}) \frac{\partial^{2}}{\partial H_{\alpha} \partial H_{\beta}}\right\} V\left(t_{1}, \mathbf{H}\right)=0 \\
0 \leqslant t_{1} \leqslant t_{\mathrm{f}}, \quad \mathbf{H} \in \bar{\Omega} .
\end{gathered}
$$

The boundary conditions associated with Eq. (21) are

$V\left(t_{1}, \Gamma_{0}\right)=$ finite,

$V\left(t_{1}, \Gamma_{\mathrm{c}}\right)=0$

and the final time condition is

$V\left(t_{\mathrm{f}}, \mathbf{H}\right)=1, \quad \mathbf{H} \in \bar{\Omega}$.

Eqs. (21) $-(24)$ are the mathematical formulation for the control problem of feedback maximization of reliability of quasi-partially integrable Hamiltonian systems. Both the optimal control law and the reliability function of optimally controlled systems can be obtained form solving these equations.

Similarly, the control problem of feedback maximization of mean first-passage time for quasi-partially integrable Hamiltonian systems can be formulated as follows. Denote mean first-passage time of controlled system (19) by $E[\tau(\mathbf{H}, \mathbf{u})]$. Define the value function as

$$
V_{1}(\mathbf{H})=\sup _{\mathbf{u} \in U} E[\tau(\mathbf{H}, \mathbf{u}],
$$

which means $V_{1}(\mathbf{H})$ is the mean first-passage time of optimally controlled system (19) under control constraint $\mathbf{u} \in U$. The following dynamical programming equation for value function (25) can be derived from the dynamical programming principle [24]:

$$
\begin{gathered}
\sup _{\mathbf{u} \in U}\left\{\left[a_{\alpha}(\mathbf{H})+\varepsilon\left\langle u_{i} \frac{\partial H_{\alpha}}{\partial P_{i}}\right\rangle\right] \frac{\partial}{\partial H_{\alpha}}+\frac{1}{2} b_{\alpha \beta}(\mathbf{H}) \frac{\partial^{2}}{\partial H_{\alpha} \partial H_{\beta}}\right\} \\
V_{1}(\mathbf{H})=-1, \quad \mathbf{H} \in \bar{\Omega} .
\end{gathered}
$$

The boundary conditions associated with this equation are

$V_{1}\left(\Gamma_{0}\right)=$ finite,

$V_{1}\left(\Gamma_{\mathrm{c}}\right)=0$.

Solving Eqs. (26) - (28) yields both the optimal control law and the mean first-passage time of optimally controlled system (19).

The optimal control law can be obtained from maximizing the left-hand side of Eq. (21) or (26) with respect to $\mathbf{u} \in U$. Suppose that the control constraints are of the form

$-b_{i} \leqslant u_{i} \leqslant b_{i}, \quad i=1,2, \ldots, n$,

where $b_{i}$ are positive constants. Then the terms $\varepsilon\left\langle u_{i}\left(\partial H_{\alpha} / \partial P_{i}\right)\right\rangle\left(\partial V / \partial H_{\alpha}\right)$ in Eqs. (21) and (26) 
will be maximum when $\left|u_{i}\right|=b_{i}$ and each term $\varepsilon u_{i}\left(\partial H_{\alpha} / \partial P_{i}\right)\left(\partial V / \partial H_{\alpha}\right)$ (summation over $\alpha$ but no summation with respect to $i$ ) is positive. Thus, the optimal control forces can be written as

$u_{i}^{*}=b_{i} \operatorname{sign}\left(\frac{\partial H_{\alpha}}{\partial P_{i}} \frac{\partial V}{\partial H_{\alpha}}\right), \quad i=1,2, \ldots, n$.

It can be shown (see Figs. 5, 6, 9 and 10) that the reliability function and mean first-passage time are monotonously decreasing functions of $H_{\alpha}$. So the value functions $V$ and $V_{1}$ should be a decreasing function of $H_{\alpha}$, i.e., $\partial V / \partial H_{\alpha}<0, \partial V_{1} / \partial H_{\alpha}<0$. Thus, if $H_{\alpha}$ contains only one $P_{\alpha}$, Eq. (30) can be reduced to

$u_{i}^{*}=-b_{i} \operatorname{sign}\left(\frac{\partial H_{i}}{\partial P_{i}}\right), \quad i=1,2, \ldots, n$,

Eq. (31) implies that the optimal control is a bangbang control. $u_{i}^{*}$ has a constant magnitude and changes its direction at $\partial H_{i} / \partial P_{i}=0$. By inserting Eq. (30) or (31) into Eq. (19) for replacing $u_{i}$ and averaging terms $\varepsilon u_{i}^{*}\left(\partial H_{\alpha} / \partial P_{i}\right)$, the following completely averaged Itô equations for optimally controlled system are obtained:

$$
\begin{aligned}
& \mathrm{d} H_{\alpha}=\bar{a}_{\alpha}(\mathbf{H}) \mathrm{d} t+\sigma_{\alpha k}(\mathbf{H}) \mathrm{d} B_{k}(t), \\
& H_{\alpha}(0)=H_{\alpha 00}, \\
& \alpha=1,2, \ldots, r, \quad k=1,2, \ldots, m,
\end{aligned}
$$

where

$$
\begin{aligned}
\bar{a}_{\alpha}(H)= & a_{\alpha}+\varepsilon\left\langle u_{i}^{*} \frac{\partial H_{\alpha}}{\partial P_{i}}\right\rangle \\
= & -\varepsilon\left\langle m_{\eta^{\prime} j} \frac{\partial H}{\partial P_{j}} \frac{\partial H_{\alpha}}{\partial P_{\eta^{\prime}}}+\varepsilon D_{k l} f_{\eta^{\prime} k} f_{\eta^{\prime \prime} l}\right. \\
& \left.\times \frac{\partial^{2} H_{\alpha}}{\partial P_{\eta^{\prime}} \partial P_{\eta^{\prime \prime}}}+\varepsilon u_{i}^{*} \frac{\partial H_{\alpha}}{\partial P_{i}}\right\rangle \\
& i, j=1,2, \ldots, n, \quad k, l=1,2, \ldots, m .
\end{aligned}
$$

Then, substituting $\bar{a}_{\alpha}(\mathbf{H})$ into Eqs. (21) and (26) to replace $a_{\alpha}(\mathbf{H})+\varepsilon u_{i} \partial H_{\alpha} / \partial P_{i}$, the final dynamical programming equations can be obtained. They are

$$
\begin{aligned}
& {\left[\frac{\partial}{\partial t_{1}}+\bar{a}_{\alpha}(\mathbf{H}) \frac{\partial}{\partial H_{\alpha}}+\frac{1}{2} b_{\alpha \beta}(\mathbf{H}) \frac{\partial^{2}}{\partial H_{\alpha} \partial H_{\beta}}\right] V\left(t_{1}, \mathbf{H}\right)} \\
& \quad=0, \quad 0 \leqslant t_{1} \leqslant t_{\mathrm{f}}, \quad \mathbf{H} \in \bar{\Omega}
\end{aligned}
$$

for the control problem of reliability maximization, and

$$
\begin{aligned}
& {\left[\bar{a}_{\alpha}(\mathbf{H}) \frac{\partial}{\partial H_{\alpha}}+\frac{1}{2} b_{\alpha \beta}(\mathbf{H}) \frac{\partial^{2}}{\partial H_{\alpha} \partial H_{\beta}}\right] V_{1}(\mathbf{H})=-1,} \\
& \mathbf{H} \in \bar{\Omega}
\end{aligned}
$$

for the control problem of maximization of mean first-passage time. The boundary conditions and final condition for Eq. (34) are still Eqs. (22)-(24) while the boundary conditions for Eq. (35) are still Eqs. (27) and (28).

\section{Relationship between dynamics programming equations and backward Kolmogorov equation and Pontryagin equation}

Denote the reliability function of optimally controlled system in time interval $[0, t]$ by $R_{\text {opt }}$. i.e.,

$R_{\text {opt }}\left(t \mid \mathbf{H}_{0}\right)=P\left\{\mathbf{H}\left(\tau, \mathbf{u}^{*}\right) \in \bar{\Omega}, \tau \in(0, t] \mid \mathbf{H}_{0} \in \bar{\Omega}\right\}$.

Following the same procedure as that leading to Eqs. (9)-(12), the following equation for $R_{\mathrm{opt}}$ can be derived:

$\frac{\partial R_{\mathrm{opt}}}{\partial t}=\bar{a}_{\alpha}\left(\mathbf{H}_{0}\right) \frac{\partial R_{\mathrm{opt}}}{\partial H_{\alpha 0}}+\frac{1}{2} b_{\alpha \beta}\left(\mathbf{H}_{0}\right) \frac{\partial^{2} R_{\mathrm{opt}}}{\partial H_{\alpha 0} \partial H_{\beta 0}}$

with boundary conditions

$R_{\text {opt }}\left(t \mid \Gamma_{0}\right)=$ finite,

$R_{\text {opt }}\left(t \mid \Gamma_{\mathrm{c}}\right)=0$

and initial condition

$R_{\text {opt }}\left(0 \mid \mathbf{H}_{0}\right)=1, \quad \mathbf{H}_{0} \in \bar{\Omega}$.

Note that in Eq. (37), $t$ is a forward time running from 0 to $t_{\mathrm{f}}$ while in Eq. (34), $t_{1}$ is a backward time running from $t_{\mathrm{f}}$ to 0 . Introducing transformation

$t=t_{\mathrm{f}}-t_{1}$.

Then Eqs. (34) and (37) will be of the same form and final time condition (24) is of the same form of initial condition (40). Note that boundary conditions (22) and (23) are of the same form of boundary conditions (38) and (39). From these and the definitions of $V\left(t_{1}, \mathbf{H}\right)$ and $R_{\text {opt }}\left(t, \mid \mathbf{H}_{0}\right)$ in Eqs. (20) and (36), respectively, it is seen that

$V\left(0, \mathbf{H}_{0}\right)=R_{\text {opt }}\left(t_{\mathrm{f}} \mid \mathbf{H}_{0}\right)$. 
Thus, we can obtain the reliability function of optimally controlled system from solving backward Kolmogorov equation (37) instead of dynamical programming equation (34). Furthermore, the probability density of first-passage time of optimally controlled system can be obtained from the following equation:

$$
\begin{aligned}
p_{\text {opt }}\left(T \mid \mathbf{H}_{0}\right) & =-\left.\frac{\partial R_{\text {opt }}\left(t \mid \mathbf{H}_{0}\right)}{\partial t}\right|_{t=T} \\
& =\left.\frac{\partial V\left(t_{1}, \mathbf{H}\right)}{\partial t_{1}}\right|_{t_{1}=0, \mathbf{H}=\mathbf{H}_{0}} .
\end{aligned}
$$

Similarly, the conditional mean of the first-passage time of optimally controlled system, i.e.,

$\mu_{1, \text { opt }}\left(\mathbf{H}_{0}\right)=\int_{0}^{\infty} T p_{\text {opt }}\left(T \mid \mathbf{H}_{0}\right) \mathrm{d} T$

is governed by the following Pontryagin equation:

$\frac{1}{2} b_{r s}\left(\mathbf{H}_{0}\right) \frac{\partial^{2} \mu_{1, \mathrm{opt}}}{\partial H_{r 0} \partial H_{s 0}}+\bar{a}_{r}\left(\mathbf{H}_{0}\right) \frac{\partial \mu_{1, \mathrm{opt}}}{\partial H_{r 0}}=-1$

together with boundary conditions

$\mu_{1, \text { opt }}\left(\Gamma_{0}\right)=$ finite,

$\mu_{1, \mathrm{opt}}\left(\Gamma_{\mathrm{c}}\right)=0$.

Obviously, dynamical programming equation (35) and boundary conditions (27) and (28) are of the same form of Eqs. (45)-(47). Thus, we have

$\mu_{1, \mathrm{opt}}\left(\mathbf{H}_{0}\right)=\left.V_{1}(\mathbf{H})\right|_{\mathbf{H}=\mathbf{H}_{0}}$.

Therefore, we can first solve dynamical programming equation (34) together with boundary conditions (22), (23) and final condition (24) to obtain $V\left(t_{1}, \mathbf{H}\right)$ and then obtain the conditional reliability function and conditional probability density of first-passage time of optimally controlled quasipartially integrable Hamiltonian system by using Eqs. (42) and (43), respectively. Or, we can directly solve backward Kolmogorov equation (37) together with boundary conditions (38), (39) and initial condition (40) to obtain the conditional reliability function of optimally controlled quasi-partially integrable Hamiltonian system and then obtain the conditional probability density of the first passage time by using Eq. (43). Note that the only difference between the backward Kolmogorov equations (9) and (37) of uncontrolled and optimally controlled systems lies in drift coefficients $a_{\alpha}$ and $\bar{a}_{\alpha}$.
Similarly, to obtain the conditional mean of first-passage time of optimally controlled quasipartially integrable Hamiltonian system, we can solve Pontryagin equation (45) together with boundary conditions (46) and (47), or first solve dynamical programming equation (35) together with boundary conditions (27), (28) and then use Eq. (48). The only difference between the Pontryagin equations (15) and (45) of uncontrolled and optimally controlled quasi-partially integrable Hamiltonian systems lies also in drift coefficients $a_{\alpha}$ and $\bar{a}_{\alpha}$.

Example. Consider the following 3-DOF quasiHamiltonian system:

$$
\begin{aligned}
\ddot{X}_{1} & +\dot{X}_{1}\left(\alpha_{10}+\alpha_{11} \dot{X}_{1}^{2}+\alpha_{12} \dot{X}_{2}^{2}+\alpha_{13} \dot{X}_{3}^{2}\right)+\omega_{1}^{2} X_{1} \\
& =W_{1}(t)+X_{1} W_{4}(t)+u_{1}, \\
\ddot{X}_{2} & +\dot{X}_{2}\left(\alpha_{20}+\alpha_{21} \dot{X}_{1}^{2}+\alpha_{22} \dot{X}_{2}^{2}+\alpha_{23} \dot{X}_{3}^{2}\right)+\frac{\partial U}{\partial X_{2}} \\
& =W_{2}(t)+X_{2} W_{5}(t)+u_{2}, \\
\ddot{X}_{3} & +\dot{X}_{3}\left(\alpha_{30}+\alpha_{31} \dot{X}_{1}^{2}+\alpha_{32} \dot{X}_{2}^{2}+\alpha_{33} \dot{X}_{3}^{2}\right)+\frac{\partial U}{\partial X_{3}} \\
& =W_{3}(t)+X_{3} W_{6}(t)+u_{3},
\end{aligned}
$$

where

$$
\begin{aligned}
U\left(X_{2}, X_{3}\right)= & \frac{1}{2}\left(\omega_{2}^{2} X_{2}^{2}+\omega_{3}^{2} X_{3}^{2}\right) \\
& +\frac{b}{4}\left(\omega_{2}^{2} X_{2}^{2}+\omega_{3}^{2} X_{3}^{2}\right)^{2},
\end{aligned}
$$

$\alpha_{i j}$ and $b>0$ are constants; $W_{k}(t)$ are independent Gaussian white noises with intensities $2 D_{k}$. The Wong-Zakai correction terms vanish for this example. The Hamiltonian associated with system (49) is thus

$H=H_{1}+H_{2}$,

where

$$
\begin{aligned}
& H_{1}=\left(\dot{X}_{1}^{2}+\omega_{1}^{2} X_{1}^{2}\right) / 2, \\
& H_{2}=\frac{1}{2}\left(\dot{X}_{2}^{2}+\dot{X}_{3}^{2}\right)+U\left(X_{2}, X_{3}\right),
\end{aligned}
$$

The Hamiltonian system with Hamiltonian (51) is of the form of Eq. (3) or (4) and it is partially integrable. So, system (49) is a quasi-partially integrable Hamiltonian system if $\alpha_{i j}, D_{k}$ and $u_{i}$ are of the order of $\varepsilon$. 
The averaged Itô equations are of the form of Eq. (19), i.e.,

$\mathrm{d} H_{1}=\left[a_{1}\left(H_{1}, H_{2}\right)+\left\langle u_{i} \frac{\partial H_{1}}{\partial P_{i}}\right\rangle\right] \mathrm{d} t+\sigma_{1 k} \mathrm{~d} B_{k}(t)$,

$\mathrm{d} H_{2}=\left[a_{2}\left(H_{1}, H_{2}\right)+\left\langle u_{i} \frac{\partial H_{2}}{\partial P_{i}}\right\rangle\right] \mathrm{d} t+\sigma_{2 k} \mathrm{~d} B_{k}(t)$,

$i=1,2 \ldots, 3, \quad k=1,2, \ldots, 6$,

where the drift and diffusion coefficients $a_{r}$ and $\sigma_{r k}(r=1,2)$ can be obtained by using formula similar to Eq. (6). They are

$$
\begin{aligned}
a_{1}= & -\alpha_{10} H_{1}-\frac{3}{2} \alpha_{11} H_{1}^{2}-\left(\alpha_{12}+\alpha_{13}\right) \\
& \times\left(H_{2}-\frac{1}{4} R^{2}-\frac{b}{12} R^{4}\right) H_{1}+D_{1}+\frac{D_{4}}{\omega_{1}^{2}} H_{1}, \\
a_{2}= & -\left(\alpha_{20}+\alpha_{21} H_{1}+\alpha_{30}+\alpha_{31} H_{1}\right) \\
& \times\left(H_{2}-\frac{1}{4} R^{2}-\frac{b}{12} R^{4}\right) \\
& -\frac{1}{8}\left(3 \alpha_{22}+\alpha_{23}+\alpha_{32}+3 \alpha_{33}\right) \\
& \times\left[4 H_{2}^{2}-8 H_{2} R^{2}\left(\frac{1}{4}+\frac{b}{12} R^{2}\right)\right. \\
& \left.+R^{4}\left(\frac{1}{3}+\frac{b^{2}}{20} R^{4}+\frac{b}{4} R^{2}\right)\right] \\
& +D_{2}+D_{3}+\frac{D_{5} R^{2}}{4 \omega_{2}^{2}}+\frac{D_{6} R^{2}}{4 \omega_{3}^{2}}, \\
b_{11}= & \sigma_{1 k} \sigma_{1 k}=2 D_{1} H_{1}+\frac{D_{4}}{\omega_{1}^{2}} H_{1}^{2}, \\
b_{22}= & \sigma_{2 k} \sigma_{2 k}=2\left(D_{2}+D_{3}\right)\left(H_{2}-\frac{1}{4} R^{2}-\frac{b}{12} R^{4}\right) \\
& +\frac{1}{2}\left(\frac{D_{5}}{\omega_{2}^{2}}+\frac{D_{6}}{\omega_{3}^{2}}\right)\left(H_{2}-\frac{1}{3} R^{2}-\frac{b}{8} R^{4}\right) R^{2},
\end{aligned}
$$

$b_{12}=\sigma_{1 k} \sigma_{2 k}=0$,

$b_{21}=\sigma_{2 k} \sigma_{1 k}=0$,

where

$R=\operatorname{sqrt}\left(\frac{\sqrt{1+4 b H_{2}}-1}{b}\right)$.

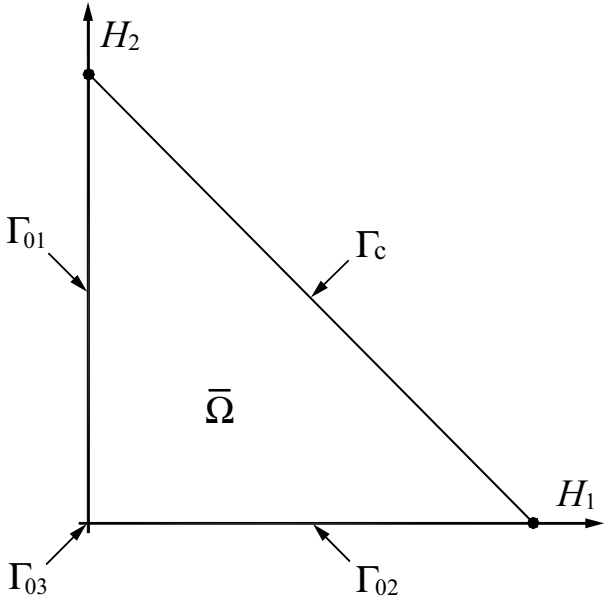

Fig. 1. Safety domain of system (49).

It is seen from Eqs. (52) and (50) that $H_{\alpha}(\alpha=1,2)$ vary from 0 to $\infty$. So, the state of averaged system (53) varies randomly in the first quadrant of plane $\left(H_{1}, H_{2}\right)$. Suppose that the limit state of system is $H=$ $H_{1}+H_{2}=H_{\mathrm{c}}$, i.e.,

$\Gamma_{\mathrm{c}}: H_{1}+H_{2}=H_{\mathrm{c}}, \quad H_{1}, H_{2} \geqslant 0$.

The safety domain $\bar{\Omega}$ of the system is the inside of a right triangle with boundaries $\Gamma_{\mathrm{c}}$ defined by Eq. (56) and $\Gamma_{0}$ defined by

$\Gamma_{0}=\Gamma_{01}+\Gamma_{02}+\Gamma_{03}$,

$\Gamma_{01}: H_{1}=0, \quad 0<H_{2}<H_{\mathrm{c}}$,

$\Gamma_{02}: H_{2}=0, \quad 0<H_{1}<H_{\mathrm{c}}$

$\Gamma_{03}: H_{1}=H_{2}=0$

(see Fig. 1).

Following Eq. (9), the conditional reliability function $R\left(t \mid H_{10}, H_{20}\right)$ of system (49) without control is governed by the following backward Kolmogorov equation:

$$
\begin{aligned}
\frac{\partial R}{\partial t}= & a_{1} \frac{\partial R}{\partial H_{10}}+a_{2} \frac{\partial R}{\partial H_{20}}+\frac{1}{2} b_{11} \frac{\partial^{2} R}{\partial H_{10}^{2}} \\
& +\frac{1}{2} b_{22} \frac{\partial^{2} R}{\partial H_{20}^{2}},
\end{aligned}
$$

where $a_{r}, b_{r r}$ are defined by Eqs. (54) with $H_{1}, H_{2}$ replaced by $H_{10}$ and $H_{20}$, respectively. The initial 
condition is Eq. (12) with $\mathbf{H}_{0}=\left[H_{10}, H_{20}\right]^{\mathrm{T}}$. One boundary condition is Eq. (11) with $\Gamma_{\mathrm{c}}$ defined by Eq. (56). The other qualitative boundary condition (10) at $\Gamma_{0}$ defined by Eq. (57) can be transformed into a quantitative one by using Eq. (58) and considering the limit behaviors of the drift and diffusion coefficients in Eq. (54) at boundary $\Gamma_{0}$. It is

$$
\frac{\partial R}{\partial t}=D_{1} \frac{\partial R}{\partial H_{10}}+a_{2}^{\prime} \frac{\partial R}{\partial H_{20}}+b_{22} \frac{\partial^{2} R}{\partial H_{20}^{2}}
$$

for boundary $\Gamma_{01}$;

$$
\frac{\partial R}{\partial t}=a_{1}^{\prime} \frac{\partial R}{\partial H_{10}}+\left(D_{2}+D_{3}\right) \frac{\partial R}{\partial H_{20}}+b_{11} \frac{\partial^{2} R}{\partial H_{10}^{2}}
$$

for boundary $\Gamma_{02}$;

$$
\frac{\partial R}{\partial t}=D_{1} \frac{\partial R}{\partial H_{10}}+\left(D_{2}+D_{3}\right) \frac{\partial R}{\partial H_{20}}
$$

for boundary $\Gamma_{03}$.

$$
\text { In Eqs. (59) }-(60)
$$

$$
\begin{aligned}
a_{1}^{\prime}= & -\alpha_{10} H_{1}-\frac{3}{2} \alpha_{11} H_{1}^{2}+D_{1}+\frac{D_{4}}{\omega_{1}^{2}} H_{1} \\
a_{2}^{\prime}= & -\left(\alpha_{20}+\alpha_{30}\right)\left(H_{2}-\frac{1}{4} R^{2}-\frac{b}{12} R^{4}\right) \\
& -\frac{1}{8}\left(3 \alpha_{22}+\alpha_{23}+\alpha_{32}+3 \alpha_{33}\right) \\
& \times\left[4 H_{2}^{2}-8 H_{2} R^{2}\left(\frac{1}{4}+\frac{b}{12} R^{2}\right)\right. \\
& \left.+R^{4}\left(\frac{1}{3}+\frac{b^{2}}{20} R^{4}+\frac{b}{4} R^{2}\right)\right] \\
& +D_{2}+D_{3}+\frac{D_{5} R^{2}}{4 \omega_{2}^{2}}+\frac{D_{6} R^{2}}{4 \omega_{3}^{2}}
\end{aligned}
$$

Eq. (58) is a two-dimensional parabolic partial differential equation and can be solved numerically together with the boundary and initial conditions by using Peaceman-Rachford scheme of finite difference method to yield the conditional reliability function of system (49) without control. The conditional probability density of the first-passage time of system (49) without control is then obtained from the conditional reliability function by using Eq. (13).

Similarly, the generalized Pontryagin equations for the conditional moments $\mu_{l+1}\left(H_{10}, H_{20}\right)$ of first passage time of system (49) without control can be derived from the averaged Itô equation (53) as follows:

$$
\begin{gathered}
\frac{1}{2} b_{11} \frac{\partial^{2} \mu_{l+1}}{\partial H_{10}^{2}}+\frac{1}{2} b_{22} \frac{\partial^{2} \mu_{l+1}}{\partial H_{20}^{2}}+a_{1} \frac{\partial \mu_{l+1}}{\partial H_{10}}+a_{2} \frac{\partial \mu_{l+1}}{\partial H_{20}} \\
=-(l+1) \mu_{l}, \quad l=1,2, \ldots,
\end{gathered}
$$

where $a_{r}, b_{r r}$ are defined by Eq. (54) with $H_{r}$ replaced by $H_{r 0}$. One boundary condition is Eq. (17) with $\Gamma_{\mathrm{c}}$ defined by Eq. (56). The other qualitative boundary condition, Eq. (16) with $\Gamma_{0}$ defined by Eq. (57), can be transformed into quantitative one by using Eq. (63) and considering the limiting behaviors of the drift and diffusion coefficients in Eq. (54) at boundary $\Gamma_{0}$. It is

$$
\begin{gathered}
\frac{1}{2} b_{22} \frac{\partial^{2} \mu_{l+1}}{\partial H_{20}^{2}}+D_{1} \frac{\partial \mu_{l+1}}{\partial H_{10}}+a_{2}^{\prime} \frac{\partial \mu_{l+1}}{\partial H_{20}} \\
=-(l+1) \mu_{l}
\end{gathered}
$$

for boundary $\Gamma_{01}$;

$$
\begin{aligned}
\frac{1}{2} & b_{11} \frac{\partial^{2} \mu_{l+1}}{\partial H_{10}^{2}}+a_{1}^{\prime} \frac{\partial \mu_{l+1}}{\partial H_{10}}+\left(D_{2}+D_{3}\right) \frac{\partial \mu_{l+1}}{\partial H_{20}} \\
= & -(l+1) \mu_{l}
\end{aligned}
$$

for boundary $\Gamma_{02}$;

$D_{1} \frac{\partial \mu_{l+1}}{\partial H_{10}}+\left(D_{2}+D_{3}\right) \frac{\partial \mu_{l+1}}{\partial H_{20}}=-(l+1) \mu_{l}$

for boundary $\Gamma_{03}$.

$a_{1}^{\prime}$ and $a_{2}^{\prime}$ in Eqs. (64) and (65) are defined by Eq. (62). Eq. (63) is a 2-dimensional elliptical partial differential equation and can be solved numerically together with boundary conditions by using five-point scheme of finite difference method to yield the conditional moments of first-passage time of system (49) without control.

Now consider the control problem of maximization of reliability of system (49). The dynamical programming equation is of the form of Eq. (21) with $\mathbf{H}=\left[H_{1}, H_{2}\right]^{\mathrm{T}}$ and with drift and diffusion coefficients, $a_{r}$ and $b_{r r}$, defined by Eq. (54). If the control forces are subject to the constraints of the form of Eq. (29), then the optimal control forces are of the form of Eq. (31). The final dynamical programming equation is of 
the form of Eq. (34), i.e.,

$$
\begin{gathered}
{\left[\frac{\partial}{\partial t_{1}}+\bar{a}_{1} \frac{\partial}{\partial H_{1}}+\bar{a}_{2} \frac{\partial}{\partial H_{2}}+\frac{1}{2} b_{11} \frac{\partial^{2}}{\partial H_{1}^{2}}\right.} \\
\left.+\frac{1}{2} b_{22} \frac{\partial^{2}}{\partial H_{2}^{2}}\right] V\left(t_{1}, H_{1}, H_{2}\right)=0,
\end{gathered}
$$

where $0 \leqslant t_{1} \leqslant t_{\mathrm{f}}, \bar{a}_{1}$ and $\bar{a}_{2}$ are obtained by using Eq. (33) as follows:

$$
\begin{aligned}
\bar{a}_{1}= & a_{1}-\frac{2 b_{1}}{\pi} \sqrt{2 H_{1}}, \\
\bar{a}_{2}^{\prime}= & a_{2}+\frac{\left(b_{2}+b_{3}\right)}{\pi R^{2}}\left[\frac{1}{b} \sqrt{R^{4}-\frac{2}{b} R^{2}}-\left(R^{2}+\frac{1}{b}\right)^{2}\right. \\
& \left.\times\left(\frac{\pi}{2}-\sin ^{-1} \frac{1}{\left(1+b R^{2}\right)}\right)\right] \sqrt{\frac{b}{2}}
\end{aligned}
$$

while $a_{1}, a_{2} b_{11}$, and $b_{22}$ are defined by Eq. (54).

One boundary condition is of the form of Eq. (23) with $\Gamma_{\mathrm{c}}$ defined by Eq. (56). The other qualitative boundary condition (22) with $\Gamma_{0}$ defined by Eq. (57) can be transformed into quantitative one by using Eq. (67) and the limiting behaviors of drift and diffusion coefficients $\bar{a}_{r}, b_{r r}$ at boundary $\Gamma_{0}$. It is

$$
\begin{gathered}
\left\{\frac{\partial}{\partial t_{1}}+D_{1} \frac{\partial}{\partial H_{1}}+\bar{a}_{2}^{\prime} \frac{\partial}{\partial H_{2}}+b_{22} \frac{\partial^{2}}{\partial H_{2}^{2}}\right\} \\
V\left(t_{1}, H_{1}, H_{2}\right)=0
\end{gathered}
$$

for boundary $\Gamma_{01}$;

$$
\begin{aligned}
& \left\{\frac{\partial}{\partial t_{1}}+\bar{a}_{1}^{\prime} \frac{\partial}{\partial H_{1}}+\left(D_{2}+D_{3}\right) \frac{\partial}{\partial H_{2}}+b_{11} \frac{\partial^{2}}{\partial H_{1}^{2}}\right\} \\
& \quad \times V\left(t_{1}, H_{1}, H_{2}\right)=0
\end{aligned}
$$

for boundary $\Gamma_{02}$;

$$
\begin{gathered}
\left\{\frac{\partial}{\partial t_{1}}+D_{1} \frac{\partial}{\partial H_{1}}+\left(D_{2}+D_{3}\right) \frac{\partial}{\partial H_{2}}\right\} \\
V\left(t_{1}, H_{1}, H_{2}\right)=0
\end{gathered}
$$

for boundary $\Gamma_{03}$.

In Eqs. (69) and (70).

$$
\bar{a}_{1}^{\prime}=a_{1}^{\prime}-\frac{2 b_{1}}{\pi} \sqrt{2 H_{1}} \text {. }
$$

$$
\begin{aligned}
\bar{a}_{2}^{\prime}= & a_{2}^{\prime}+\frac{\left(b_{2}+b_{3}\right)}{\pi R^{2}}\left[\frac{1}{b} \sqrt{R^{4}-\frac{2}{b} R^{2}}-\left(R^{2}+\frac{1}{b}\right)^{2}\right. \\
& \left.\times\left(\frac{\pi}{2}-\sin ^{-1} \frac{1}{\left(1+b R^{2}\right)}\right)\right] \sqrt{\frac{b}{2}}
\end{aligned}
$$

while $a_{1}^{\prime}$ and $a_{2}^{\prime}$ are defined by Eq. (62).

As indicated in the last section, dynamical programming equation (67) with boundary conditions (23), (69)-(71) and final condition (24) is equivalent to the following backward Kolmogorov equation for $R_{\text {opt }}\left(t, \mathbf{H}_{0}\right)$

$$
\begin{aligned}
\frac{\partial R_{\mathrm{opt}}}{\partial t}= & \bar{a}_{1} \frac{\partial R_{\mathrm{opt}}}{\partial H_{10}}+\bar{a}_{2} \frac{\partial R_{\mathrm{opt}}}{\partial H_{20}}+\frac{1}{2} b_{11} \frac{\partial^{2} R_{\mathrm{opt}}}{\partial H_{10}^{2}} \\
& +\frac{1}{2} b_{22} \frac{\partial^{2} R_{\mathrm{opt}}}{\partial H_{20}^{2}}
\end{aligned}
$$

with initial condition (40), boundary condition (39) with $\Gamma_{\mathrm{c}}$ defined by Eq. (56) and boundary conditions

$\frac{\partial R_{\mathrm{opt}}}{\partial t}=D_{1} \frac{\partial R_{\mathrm{opt}}}{\partial H_{10}}+\bar{a}_{2}^{\prime} \frac{\partial R_{\mathrm{opt}}}{\partial H_{20}}+\frac{1}{2} b_{22} \frac{\partial^{2} R_{\mathrm{opt}}}{\partial H_{20}^{2}}$

for boundary $\Gamma_{01}$;

$$
\begin{aligned}
\frac{\partial R_{\mathrm{opt}}}{\partial t}= & \bar{a}_{1}^{\prime} \frac{\partial R_{\mathrm{opt}}}{\partial H_{10}}+\left(D_{2}+D_{3}\right) \frac{\partial R_{\mathrm{opt}}}{\partial H_{20}} \\
& +\frac{1}{2} b_{11} \frac{\partial^{2} R_{\mathrm{opt}}}{\partial H_{10}^{2}}
\end{aligned}
$$

for boundary $\Gamma_{02}$;

$\frac{\partial R_{\mathrm{opt}}}{\partial t}=D_{1} \frac{\partial R_{\mathrm{opt}}}{\partial H_{10}}+\left(D_{2}+D_{3}\right) \frac{\partial R_{\mathrm{opt}}}{\partial H_{20}}$

for boundary $\Gamma_{03}$.

Since the backward Kolmogorov Eq. (73) and its initial and boundary conditions for optimally controlled system (49) are of the same form of the backward Kolmogorov equation (58) and its initial and boundary conditions for system (49) without control except drift coefficients, it is more convenient to obtain the conditional reliability function $R_{\text {opt }}\left(t \mid H_{10}, H_{20}\right)$ of optimally controlled system (49) from solving backward Kolmogorov equation (73) together with its initial and boundary conditions.

For the control problem of maximization of mean first-passage time of system (49), we can formulate the dynamical programming equation and its equivalent Pontryagin equation similarly. The dynamical 
programming equation is

$$
\begin{aligned}
& \left\{\bar{a}_{1} \frac{\partial}{\partial H_{1}}+\bar{a}_{2} \frac{\partial}{\partial H_{2}}+\frac{1}{2} b_{11} \frac{\partial^{2}}{\partial H_{1}^{2}}+\frac{1}{2} b_{22} \frac{\partial^{2}}{\partial H_{2}^{2}}\right\} \\
& \quad \times V_{1}\left(H_{1}, H_{2}\right)=-1
\end{aligned}
$$

with boundary condition (28), where $\Gamma_{\mathrm{c}}$ is defined by Eq. (56), and boundary conditions

$$
\begin{aligned}
& \left\{D_{1} \frac{\partial}{\partial H_{1}}+\bar{a}_{2}^{\prime} \frac{\partial}{\partial H_{2}}+\frac{1}{2} b_{22} \frac{\partial^{2}}{\partial H_{2}^{2}}\right\} \\
& \times V_{1}\left(H_{1}, H_{2}\right)=-1
\end{aligned}
$$

for boundary $\Gamma_{01}$;

$$
\begin{aligned}
& \left\{\bar{a}_{1}^{\prime} \frac{\partial}{\partial H_{1}}+\left(D_{2}+D_{3}\right) \frac{\partial}{\partial H_{2}}+\frac{1}{2} b_{11} \frac{\partial^{2}}{\partial H_{1}^{2}}\right\} \\
& \quad \times V_{1}\left(H_{1}, H_{2}\right)=-1
\end{aligned}
$$

for boundary $\Gamma_{02}$;

$$
\left\{D_{1} \frac{\partial}{\partial H_{1}}+\left(D_{2}+D_{3}\right) \frac{\partial}{\partial H_{2}}\right\} V_{1}\left(H_{1}, H_{2}\right)=-1
$$

for boundary $\Gamma_{03}$.

The equivalent Pontryagin equation is

$$
\begin{aligned}
\frac{1}{2} b_{11} \frac{\partial^{2} \mu_{1, \mathrm{opt}}}{\partial H_{10}^{2}}+\frac{1}{2} b_{22} \frac{\partial^{2} \mu_{1, \mathrm{opt}}}{\partial H_{20}^{2}} \\
+\bar{a}_{1} \frac{\partial \mu_{1, \mathrm{opt}}}{\partial H_{10}}+\bar{a}_{2} \frac{\partial \mu_{1, \mathrm{opt}}}{\partial H_{20}}=-1
\end{aligned}
$$

with boundary condition (47), where $\Gamma_{\mathrm{c}}$ is defined by Eq. (56), and boundary conditions

$\frac{1}{2} b_{22} \frac{\partial^{2} \mu_{1, \mathrm{opt}}}{\partial H_{20}^{2}}+D_{1} \frac{\partial \mu_{1, \mathrm{opt}}}{\partial H_{10}}+\bar{a}_{2}^{\prime} \frac{\partial \mu_{1, \mathrm{opt}}}{\partial H_{20}}=-1$

for boundary $\Gamma_{01}$;

$$
\begin{aligned}
& \frac{1}{2} b_{11} \frac{\partial^{2} \mu_{1, \mathrm{opt}}}{\partial H_{10}^{2}}+\bar{a}_{1}^{\prime} \frac{\partial \mu_{1, \mathrm{opt}}}{\partial H_{10}}+\left(D_{2}+D_{3}\right) \frac{\partial \mu_{1, \mathrm{opt}}}{\partial H_{20}} \\
& =-1
\end{aligned}
$$

for boundary $\Gamma_{02}$;

$D_{1} \frac{\partial \mu_{1, \mathrm{opt}}}{\partial H_{10}}+\left(D_{2}+D_{3}\right) \frac{\partial \mu_{1, \mathrm{opt}}}{\partial H_{20}}=-1$

for boundary $\Gamma_{03}$. It is also more convenient to obtain the mean first-passage time of optimally controlled system (49) from solving the Pontryagin equation (81) with its boundary conditions.

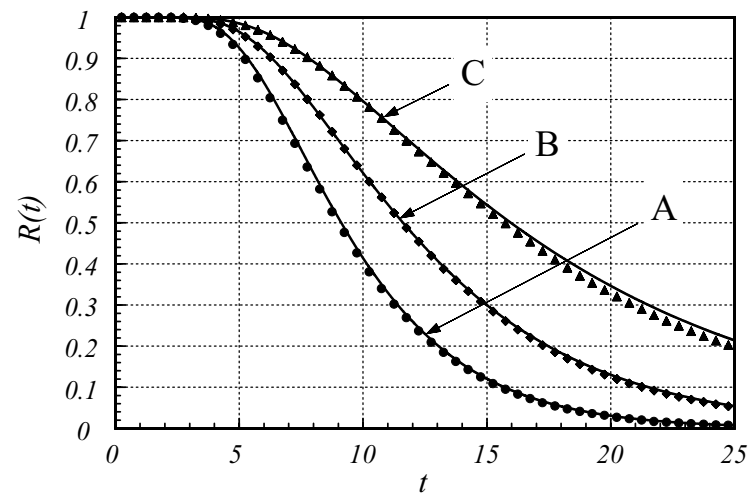

Fig. 2. Reliability function of system (49): (A) uncontrolled; (B) optimally controlled with $b_{1}=0.01, b_{2}=0.02$, and $b_{3}=0.03$; and (C) optimally controlled with $b_{1}=0.02, b_{2}=0.04$, and $b_{3}=0.06$. (一) Analytical result; $(\bullet, \bullet, \mathbf{\Delta})$ from digital simulation.

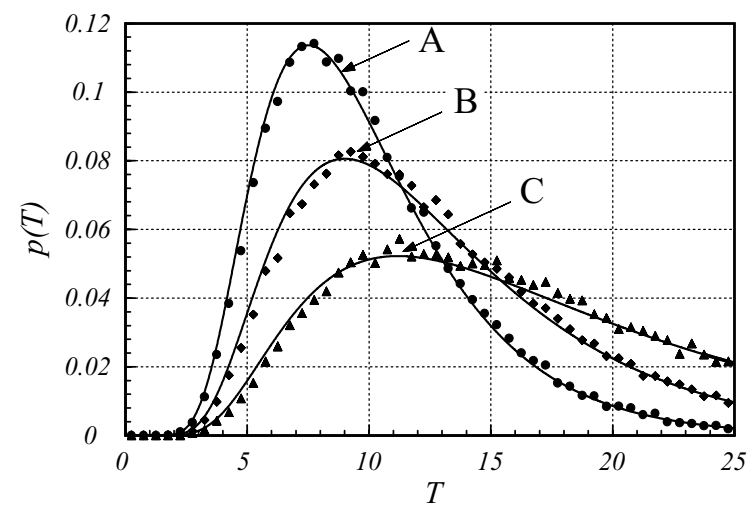

Fig. 3. Probability density of first-passage time of system (49): (A) uncontrolled; (B) optimally controlled with $b_{1}=0.01, b_{2}=0.02$, and $b_{3}=0.03$; and (C) optimally controlled with $b_{1}=0.02, b_{2}=0.04$, and $b_{3}=0.06$. (-) Analytical result; $(\bullet, \bullet, \mathbf{\Delta})$ from digital simulation.

Some numerical results for the reliability functions, probability density and mean of first-passage time of uncontrolled and optimally controlled system (49) are shown in Figs. 2-10. In the calculation, system parameters are set up as follows:

$\alpha_{10}=\alpha_{20}=\alpha_{30}=-0.08, \quad \alpha_{11}=\alpha_{22}=\alpha_{33}=0.04$,

$\alpha_{12}=\alpha_{13}=0.01, \quad \alpha_{21}=\alpha_{31}=0.02$,

$\alpha_{23}=\alpha_{32}=0.04$,

$\omega_{1}=1.414, \quad \omega_{2}=1, \quad \omega_{3}=1.732, \quad b=1$, 


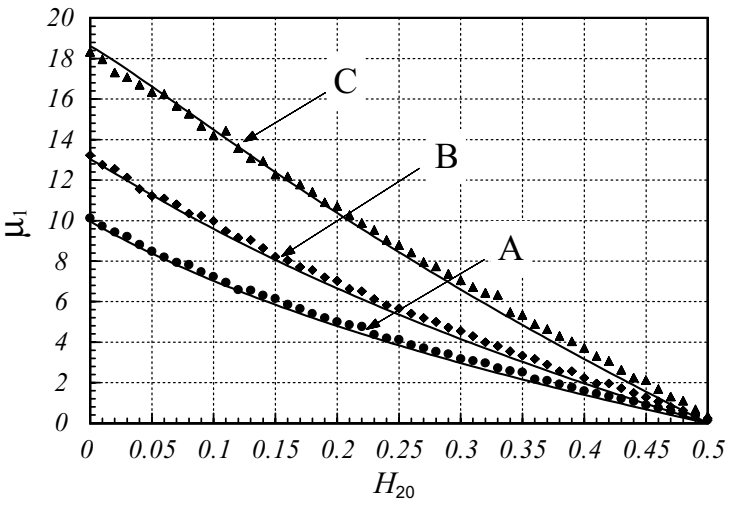

Fig. 4. Mean first-passage time of system (49): (A) uncontrolled; (B) optimally controlled with $b_{1}=0.01, b_{2}=0.02$, and $b_{3}=0.03$; and (C) optimally controlled with $b_{1}=0.02, b_{2}=0.04$, and $b_{3}=0.06$. $(-)$ Analytical result; $(\bullet, \bullet, \mathbf{\Delta})$ from digital simulation.

$2 D_{1}=0.01,2 D_{2}=0.02,2 D_{3}=0.03,2 D_{4}=0.1,2 D_{5}=$ $0.2,2 D_{6}=0.3 . \Gamma_{\mathrm{c}}$ is $H_{\mathrm{c}}=H_{1}+H_{2}=0.5$. The initial condition of the system is at $\Gamma_{03}$, i.e., $H_{10}=H_{20}=0$. Note that $\Gamma_{03}$ is a reflecting boundary. So it can be taken as initial condition.
In Figs. 2-4, solid line represents the analytical results by using proposed procedures while symbols $\bullet$, $\checkmark$ and $\boldsymbol{\Delta}$ those from digital simulation. It is seen from Figs. 2-4 that the two results are in excellent agreement. It is also seen from these figures that both the reliability and mean first-passage time can indeed be raised by feedback control. This last conclusion can also be drawn from comparisons of Figs. 5 and 6, 7 and 8 , and 9 and 10 . Besides, one more conclusion can be drawn from comparisons of Figs. 5 and 6, and 9 and 10 that both reliability function and mean first-passage time of uncontrolled and optimally controlled system (49) are monotonously decreasing function of initial energies $H_{10}$ and $H_{20}$. This justifies the derivation from Eq. (30) to (31).

It is noted that the proposed procedures are applicable to any shape of safety domain $\bar{\Omega}$. For example, suppose that only $H_{1}$ is critical while $H_{2}$ is of no concern. In this case, the safety domain $\bar{\Omega}$ is shown in Fig. 11. The boundary of safety domain consists of $\Gamma_{\mathrm{c}}$ and $\Gamma_{0}$, where

$\Gamma_{\mathrm{c}}: H_{1}=H_{\mathrm{c}}, \quad 0 \leqslant H_{2}<\infty$

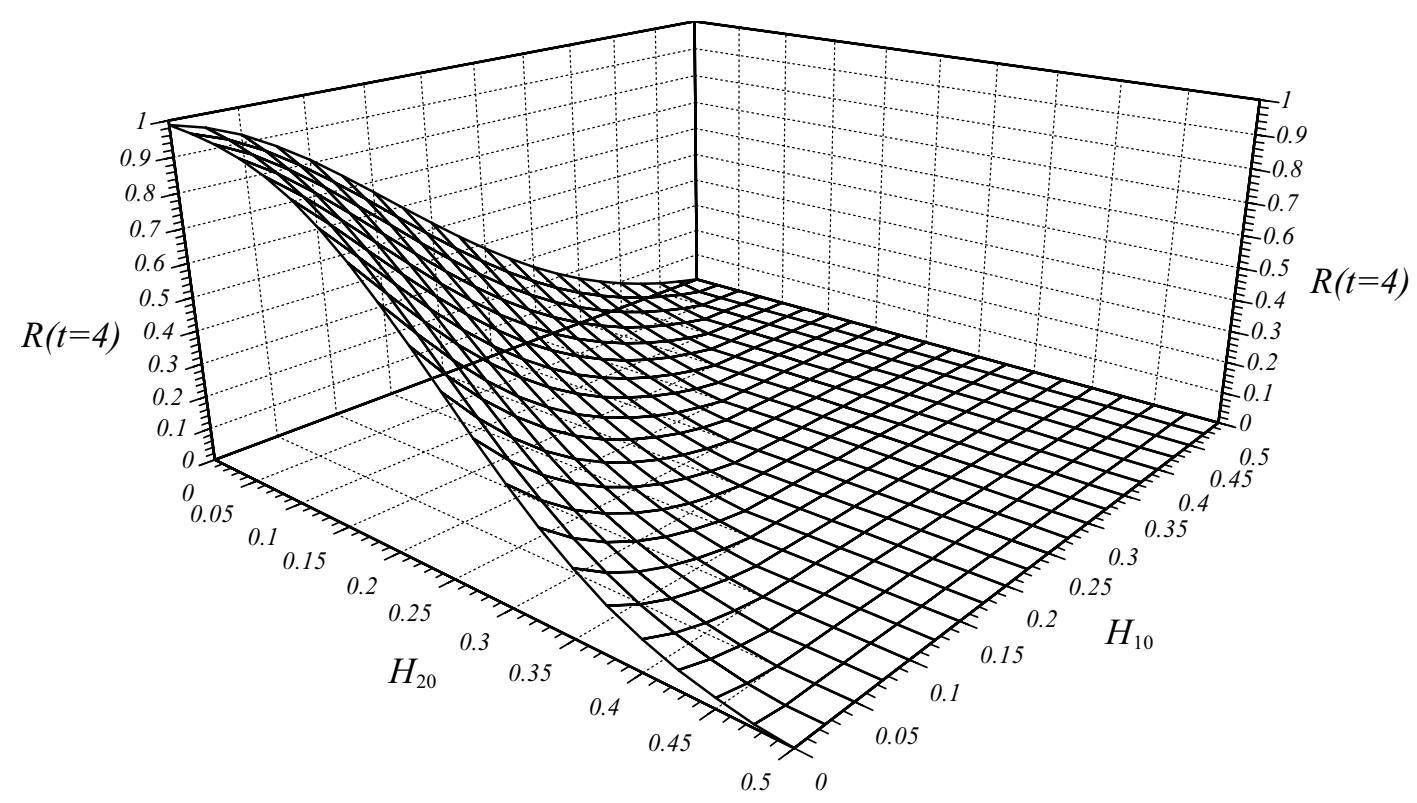

Fig. 5. Reliability as function of $H_{10}$ and $H_{20}$ of uncontrolled system (49). 


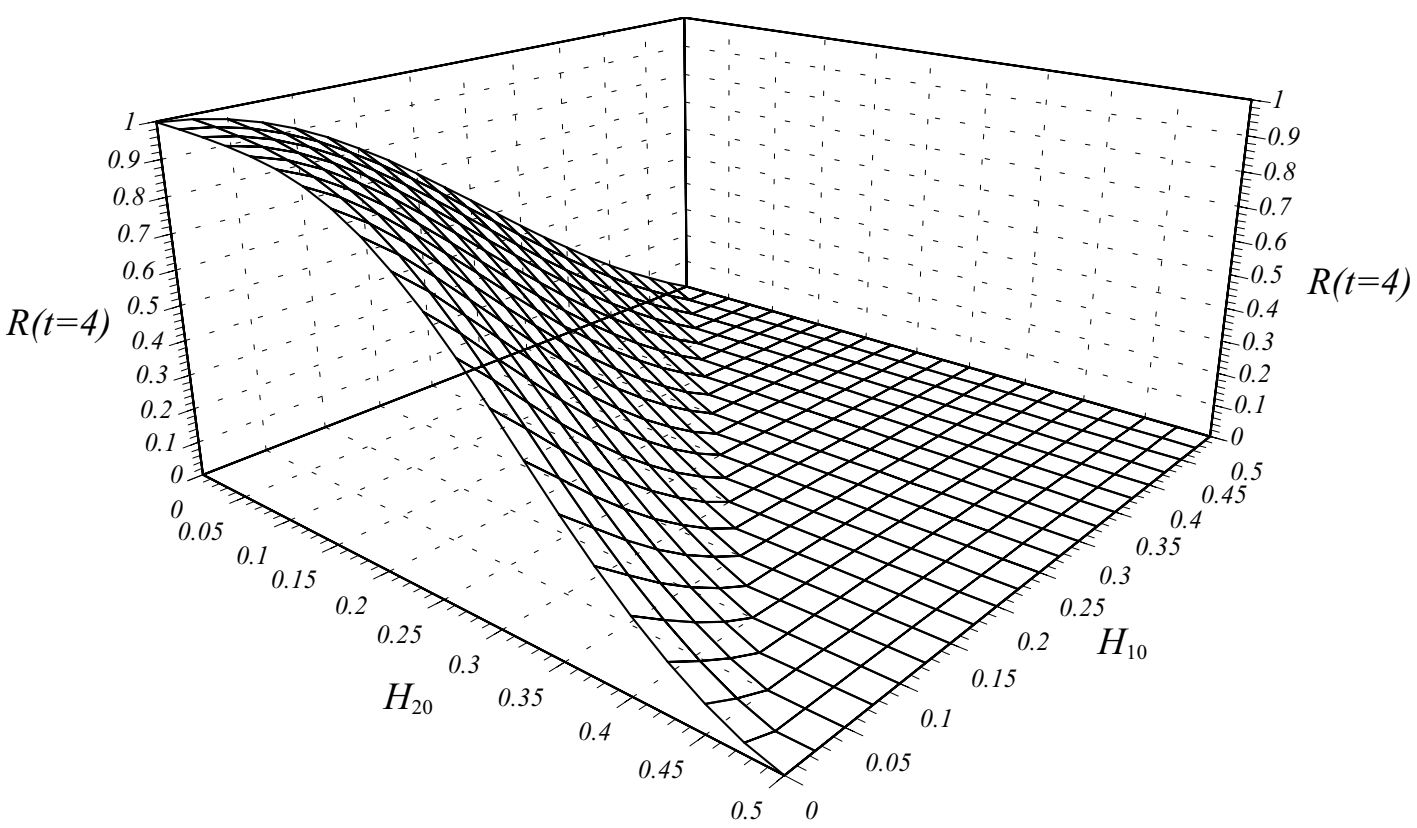

Fig. 6. Reliability as function of $H_{10}$ and $H_{20}$ of optimally controlled system (49) with $b_{1}=0.02, b_{2}=0.04$, and $b_{3}=0.06$.

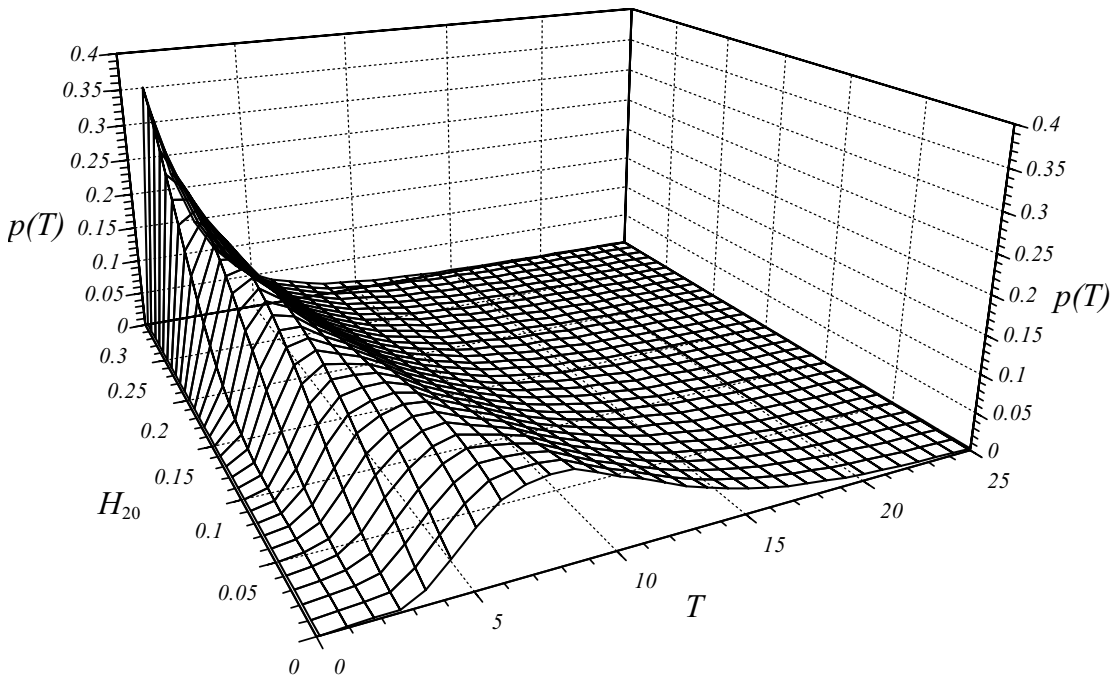

Fig. 7. Probability density of first-passage time as function of $H_{20}$ and $T$ of uncontrolled system (49).

and

$\Gamma_{0}=\Gamma_{01}+\Gamma_{02}+\Gamma_{03}$,

$\Gamma_{01}: H_{1}=0, \quad 0<H_{2}<\infty$,
$\Gamma_{02}: H_{2}=0, \quad 0<H_{1}<H_{\mathrm{c}}$,

$\Gamma_{03}: H_{1}=H_{2}=0$.

All the equations from (53) to (84) hold and can be solved similarly. 


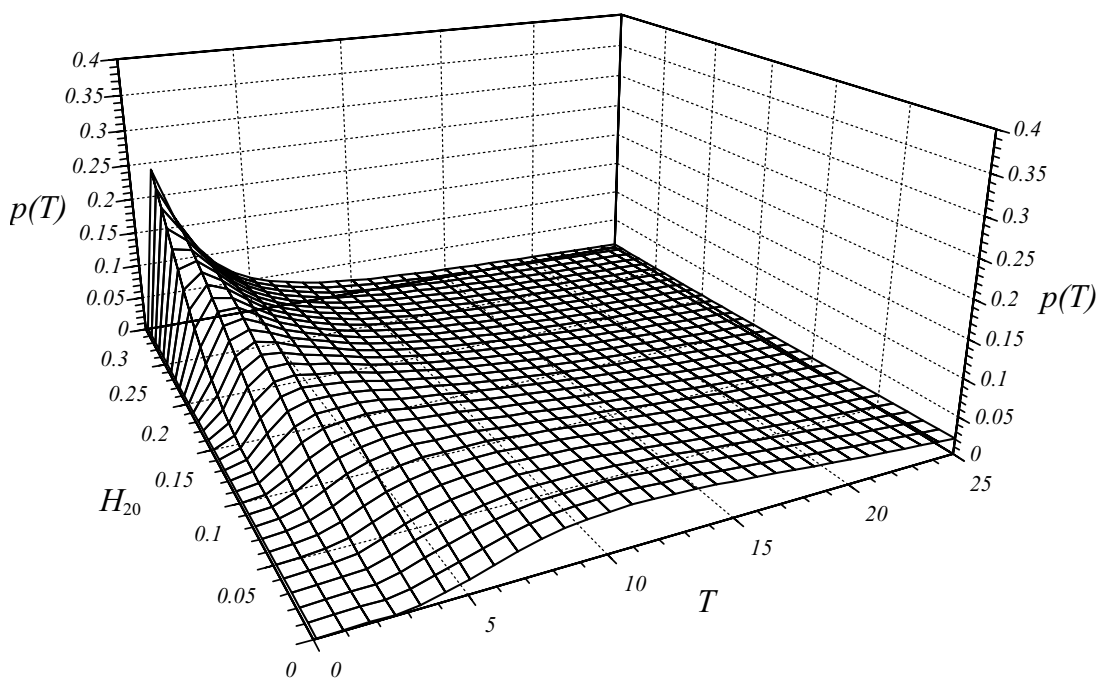

Fig. 8. Probability density of first-passage time as function of $H_{20}$ and $T$ of optimally controlled system $(49)$ with $b_{1}=0.02, b_{2}=0.04$, and $b_{3}=0.06$.

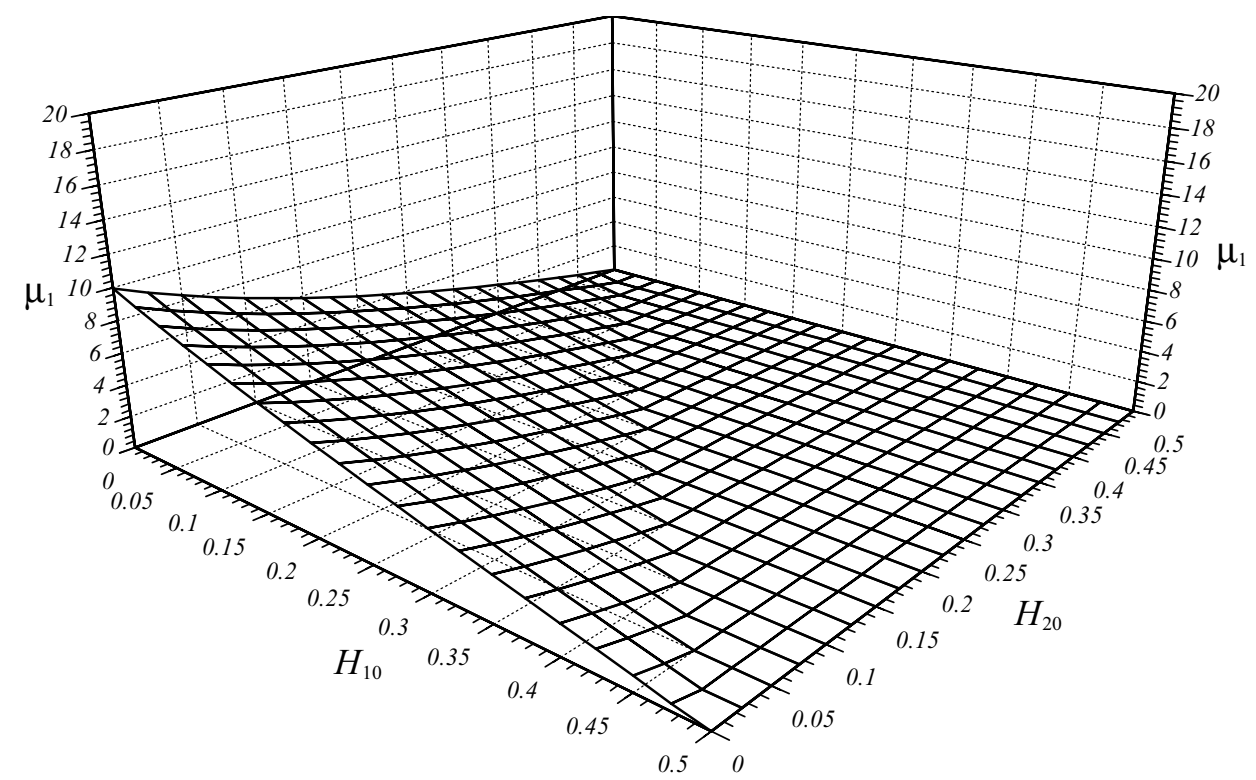

Fig. 9. Mean first-passage time as function of $H_{10}$ and $H_{20}$ of uncontrolled system (49).

\section{Conclusions}

In the present paper, the procedures for studying the first-passage failure and its feedback minimization of quasi-partially integrable Hamiltonian systems have been proposed based on the stochastic averaging method for quasi-partially integrable Hamiltonian systems. For uncontrolled systems, the approximate statistics of the first-passage failure are obtained from solving the backward Kolmogorov equation for the 


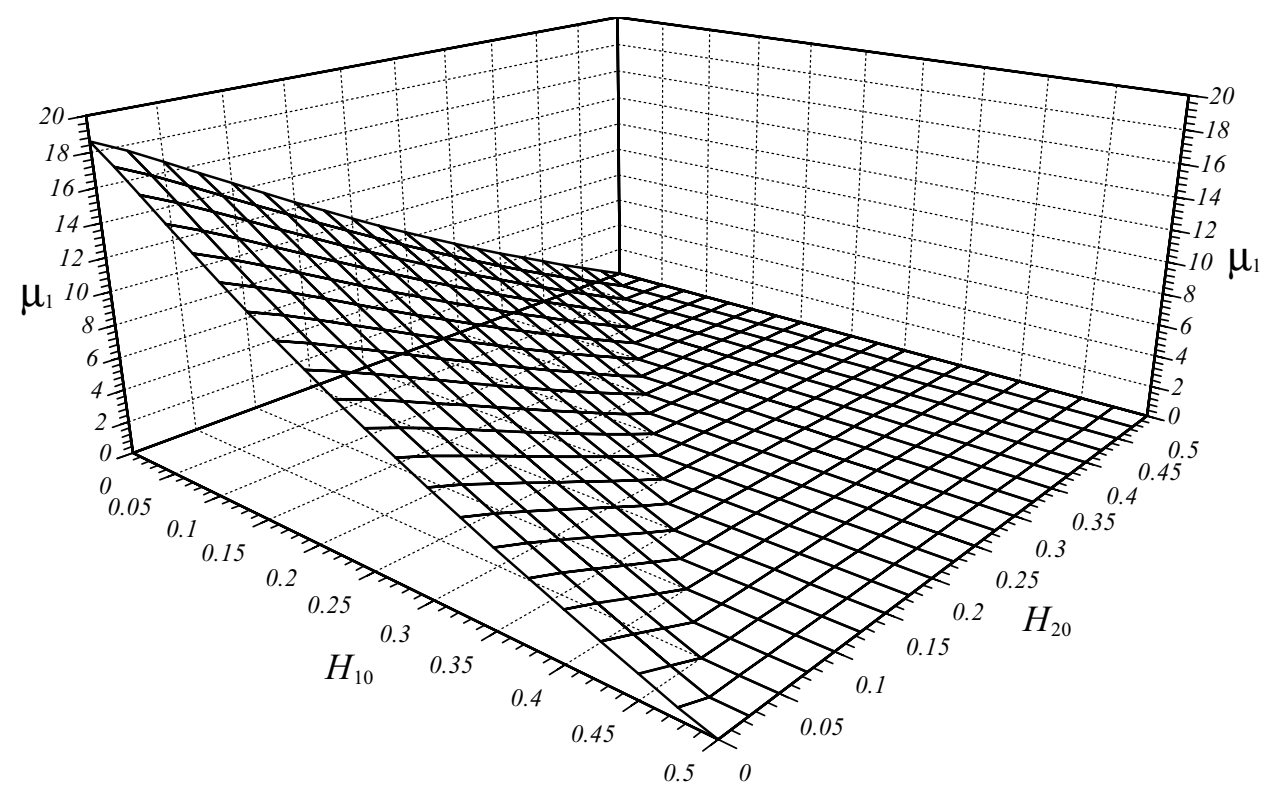

Fig. 10. Mean first-passage time as function of $H_{10}$ and $H_{20}$ of optimally controlled system (49) with $b_{1}=0.02, b_{2}=0.04$, and $b_{3}=0.06$.

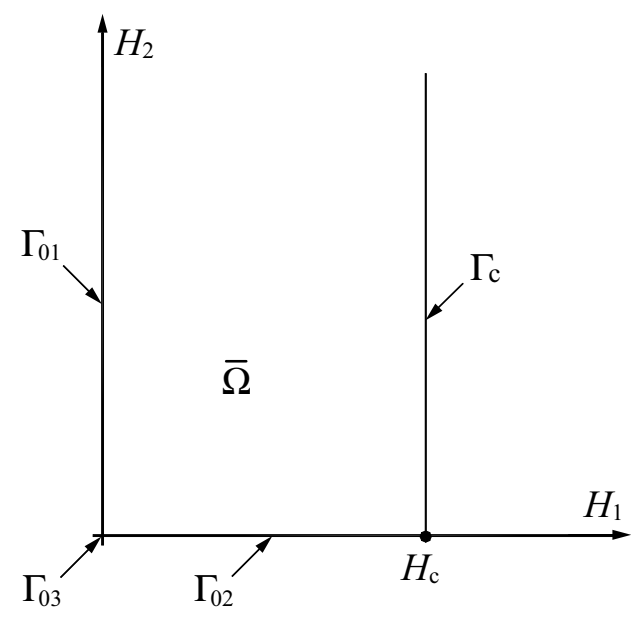

Fig. 11. Safety domain of system (49) when only $H_{1}$ is critical while $\mathrm{H}_{2}$ is of no concern.

conditional reliability function and/or the generalized Pontryagin equations for the conditional moments of first-passage time of averaged Itô equations. For controlled systems, the optimally control law is obtained from solving the dynamical programming equations for maximization of reliability and/or for maximization of mean first-passage time of averaged
Itô equations, while the approximate statistics of the first-passage failure of optimally controlled systems are obtained from solving the final dynamical programming equations of completely averaged Itô equations or from solving their equivalent backward Kolmogorov equation and Pontryagin equation. An example has been given to illustrate the application of the procedures. From the numerical results for the example, the following conclusions can be made:

1. The proposed procedures may yield quite accurate result;

2. The first-passage failure can be reduced significantly by feedback control;

3. The reliability and mean first-passage time of uncontrolled and controlled systems are monotonously decreasing function of initial first integrals.

\section{Acknowledgements}

The work reported in this paper was supported by the National Natural Science Foundation of China under Grants No. 19972059 and 10002015 and the Special Fund for Doctor Programs in Institutions of Higher Learning of China. 


\section{References}

[1] A.T. Bharucha-Reid, Elements of Markov Processes and their Applications, McGraw-Hill, New York, 1960.

[2] D.R. Cox, H.D. Miller, The Theory of Stochastic Processes, Chapman and Hall, New York, 1965.

[3] S.T. Ariaratnam, H.N. Pi, On the first-passage time for envelope crossing for a linear oscillator, Int. J. Control 18 (1973) 89-96.

[4] W.C. Lennox, D.A. Fraser, On the first passage distribution for the envelope of a non-stationary narrow-band stochastic process, J. Appl. Mech. ASME 41 (1974) 793-797.

[5] S.T. Ariaratnam, D.S.F. Tam, Random vibration and stability of a linear parametrically excited oscillator, Z. Angew. Math. Mech. 59 (1979) 79-84.

[6] P.D. Spanos, G.P. Solomos, Barrier crossing due to transient excitation, J. Eng. Mech. Div. ASCE 110 (1984) 20-36.

[7] J.B. Roberts, First passage probability for nonlinear oscillator, J. Eng. Mech. Div. ASCE 102 (1976) 851-866.

[8] J.B. Roberts, First-passage time for oscillator with nonlinear restoring forces, J. Sound Vib. 56 (1978) 71-86.

[9] J.B. Roberts, Response of an oscillator with nonlinear damping and a softening spring to non-white random excitation, Prob. Eng. Mech. 1 (1986) 40-48.

[10] J.B. Roberts, First-passage time for randomly excited nonlinear oscillator, J. Sound Vib. 109 (1986) 33-50.

[11] P.D. Spanos, Survival probability of non-linear oscillators subjected to broad-band random disturbance, Int. J. Non-Linear Mech. 17 (1982) 303-317.

[12] W.Q. Zhu, Y. Lei, First passage time for state transition of randomly excited systems, Proceedings of the 47th Session of International Statistical Institute, Vol. LIII, Book 3, 1989, pp. 517-531 (Invited Papers).

[13] G.Q. Cai, Y.K. Lin, On statistics of first-passage failure, J. Appl. Mech. ASME 61 (1994) 93-99.
[14] W.Q. Zhu, Y.Q. Yang, Stochastic averaging of quasi-non-integrable-Hamiltonian systems, J. Appl. Mech. ASME 64 (1997) 157-164.

[15] W.Q. Zhu, Z.L. Huang, Y.Q. Yang, Stochastic averaging of quasi-integrable Hamiltonian systems, J. Appl. Mech. ASME 64 (1997) 975-984.

[16] C.B. Gan, W.Q. Zhu, First-passage failure of quasi-non-integrable-Hamiltonian systems, Int. J. Non-Linear Mech. 36 (2001) 209-220.

[17] W.Q. Zhu, M.L. Deng, Z.L. Huang, First-passage failure of quasi integrable Hamiltonian systems, J. Appl. Mech. ASME, 69 (2000), in press.

[18] W.Q. Zhu, Z.G. Ying, Optimal nonlinear feedback control of quasi-Hamiltonian systems, Sci. China (Ser. A) 42 (1999) 1213-1219.

[19] W.Q. Zhu, Z.G. Ying, T.T. Soong, An optimal nonlinear feedback control strategy for randomly excited structural systems, Nonlinear Dyn. 24 (2001) 31-51.

[20] W.Q. Zhu, Z.L. Huang, M.L. Deng, Feedback minimization of first-passage failure of quasi non-integrable Hamiltonian systems, Int. J. Non-Linear Mech., 37 (2002) 1057-1071.

[21] W.Q. Zhu, Z.L. Huang, M.L. Deng, Feedback minimization of first-passage failure of quasi integrable Hamiltonian systems, Optional Control Applications and Methods, submitted for publication.

[22] W.Q. Zhu, Z.G. Ying, Y.Q. Ni, J.M. Ko, Optimal nonlinear stochastic control of hysteretic systems, J. Eng. Mech. ASCE 126 (2000) 1027-1032.

[23] W.Q. Zhu, Z.L. Huang, Y. Suzuki, Stochastic averaging and Lyapunov exponent of quasi partially integrable Hamiltonian system, Int. J. Non-Linear Mech. 37 (2002) 419-437.

[24] V.N. Afanas'ev, V.B. Kolmanovski, V.R. Nosov, Mathematical Theory of Control System Design, Kluwer Academic Publishers, Dordrecht, 1996. 